\title{
Review of nemonoxacin with special focus on clinical development
}

This article was published in the following Dove Press journal:

Drug Design, Development and Therapy

5 July 2014

Number of times this article has been viewed

\section{Xiaohua Qin \\ Haihui Huang}

Institute of Antibiotics, Huashan Hospital, Fudan University, Shanghai, People's Republic of China

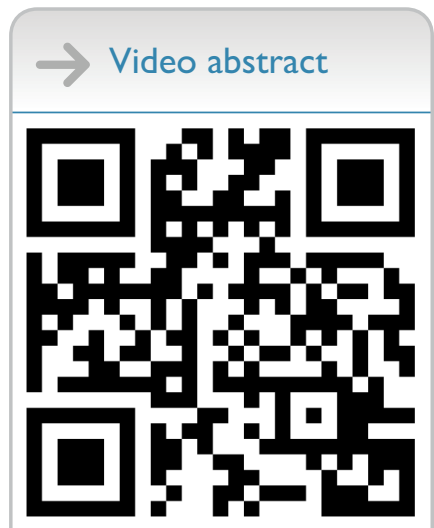

Point your SmartPhone at the code above. If you have a QR code reader the video abstract will appear. Or use: http://dvpr.es/lionW3q
Correspondence: Haihui Huang Institute of Antibiotics, Huashan Hospital, Fudan University, Shanghai 200040,

People's Republic of China

Tel +86 2l 52888195

Email drhaihuihuang@I63.com
Abstract: Nemonoxacin is a novel C-8-methoxy nonfluorinated quinolone with remarkably enhanced in vitro activity against a wide variety of clinically relevant pathogens, especially gram-positive bacteria, including multidrug-resistant Streptococcus pneumoniae and methicillinresistant Staphylococcus aureus. It has a low propensity for selecting resistant pathogens than fluoroquinolones, since bacteria become resistant to nemonoxacin only when three different mutations occur in their quinolone resistance-determining regions. Nemonoxacin shows greater efficacy than most of the widely used fluoroquinolones in the murine model of systemic, pulmonary, or ascending urinary tract infection. Nemonoxacin has a sound PK profile in healthy volunteers. It rapidly reaches maximum concentration $\mathrm{C}_{\max } 1-2$ hours after oral administration in the fasting state and has a relatively long elimination half-life of more than 10 hours, which is similar to fluoroquinolones. Approximately $60 \%-75 \%$ of the administered dose is excreted in unchanged form via kidneys over 24-72 hours. Phase II and III studies of oral nemonoxacin and Phase II studies of intravenous nemonoxacin have been completed in patients with community-acquired pneumonia (CAP), before which the Phase I studies of oral and intravenous nemonoxacin indicated sound tolerance and safety with healthy volunteers. The published results demonstrate that an oral dose of either $500 \mathrm{mg}$ or $750 \mathrm{mg}$ nemonoxacin once daily for 7 days is as effective and safe as levofloxacin $500 \mathrm{mg}$ once daily for 7 days. Nemonoxacin is well-tolerated in patients with CAP. The most common adverse events of oral administration are observed in the gastrointestinal and nervous system, the incidence of which is similar to levofloxacin treatment. The Phase III studies of intravenous nemonoxacin for treating CAP and oral nemonoxacin for diabetic foot infection has been registered with promising outcomes to be expected.

Keywords: nemonoxacin, pharmacodynamics, pharmacokinetics, community acquired pneumonia, clinical development

\section{Introduction}

The problem of fluoroquinolone resistance, both among gram-positive cocci and gram-negative bacilli, has brought great challenge into clinical practice. ${ }^{1}$ Therefore, the development of newer quinolone compounds with greater activity and less resistance is urgently required. ${ }^{2}$

Nemonoxacin (TG-873870) is a novel C-8-methoxy nonfluorinated quinolone, which is currently under clinical development. The addition of a methoxy group at the C-8 position (Figure 1) enables nemonoxacin to target both topoisomerase IV and topoisomerase II, associated with an improved spectrum of activity and reduced mutant selection. ${ }^{3}$ On the other hand, it is considered that the removal of the fluorine residue may reduce the incidence of toxic side effects. ${ }^{4}$ 


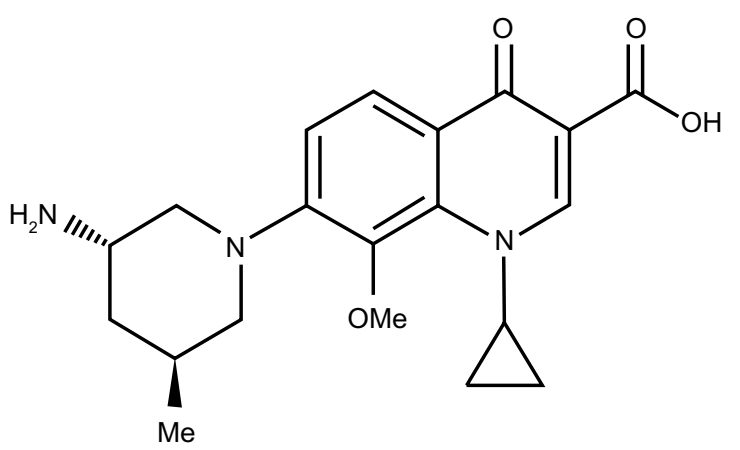

Figure I Nemonoxacin chemical structure.

Note: Antimicrob Agents Chemother, 2009;53(II):4915-4920, doi:I0.1 I28/AAC. 00078-09, amended with permission from American Society for Microbiology. ${ }^{4}$ Abbreviation: Me, methyl group.

Nemonoxacin was first developed by Procter and Gamble Pharmaceuticals (Cincinnati, OH, USA), which completed the Phase IA single-dose escalation studies in October 2004. Thereafter, it was authorized to TaiGen Biotechnology Co, Ltd (Taipei, Taiwan) for worldwide clinical trials and further development since June 2005. (http://www.taigenbiotech. com.tw/NewsDetail/215b4b8fe317484a9faf4180a95c01ce).

Up to now, TaiGen Biotechnology has completed the multidose, dose-escalation Phase IB trials in healthy volunteers and Phase II studies of oral and intravenous nemonoxacin in Chinese patients with community-acquired pneumonia (CAP). A Phase III study of intravenous nemonoxacin for treating CAP and oral nemonoxacin for diabetic foot infection have been registered.

Here, we present a comprehensive picture of nemonoxacin under clinical development by reviewing the data available on the pharmacodynamics (PD), the pharmacokinetics (PK), and the clinical treatment studies of the antimicrobial agent.

\section{PD}

\section{In vitro activity of nemonoxacin}

Based on the data reported in the previous in vitro susceptibility testing (three studies that included 2,683 strains of gram-positive cocci and 1,303 gram-negative bacilli), nemonoxacin showed potent broad-spectrum activities against most gram-positive cocci and gram-negative bacilli (Table 1). Especially, nemonoxacin demonstrated better activity than the fluoroquinolone comparators against some typical antibiotic-resistant gram-positive organisms, like penicillin-resistant Streptococcus pneumoniae (PRSP) and fluoroquinolone-resistant S. pneumoniae. ${ }^{46}$ Nemonoxacin also displayed good in vitro activities against atypical pathogens, such as Chlamydia trachomatis and C. pneumoniae. ${ }^{7}$
In a Canadian report, the activity of nemonoxacin against S. pneumoniae was superior to the fluoroquinolones tested, including penicillin-resistant $S$. pneumoniae. ${ }^{4}$ In another study, Chen et $\mathrm{al}^{5}$ found that among the $150 \mathrm{~S}$. pneumoniae isolates, nemonoxacin had comparable activity (minimum inhibitory concentration $[\mathrm{MIC}]_{90} 0.06 \mathrm{mg} / \mathrm{L}$ ) as gemifloxacin and much greater activity than that of levofloxacin $\left(\mathrm{MIC}_{90}\right.$ $2 \mathrm{mg} / \mathrm{L}$ ) and moxifloxacin. Moreover, for levofloxacin nonsusceptible (MIC $\geq 4 \mathrm{mg} / \mathrm{L}$ ) S. pneumoniae isolates, nemonoxacin successfully inhibited $76.7 \%$ (23/30) of the isolates at concentration of $\leq 0.5 \mathrm{mg} / \mathrm{L}$, while moxifloxacin inhibited $6.7 \%$ and gemifloxacin $90 \%$ at the same concentration.

Lauderdale et $\mathrm{al}^{6}$ reported lower nemonoxacin $\mathrm{MIC}_{90}$ (2 mg/L) among levofloxacin-resistant S. pneumoniae isolates (MIC $\geq 8 \mathrm{mg} / \mathrm{L}$ ) in contrast to moxifloxacin $(8 \mathrm{mg} / \mathrm{L}$ ), comparable to ceftriaxone $(2 \mathrm{mg} / \mathrm{L})$, but higher than linezolid $(1 \mathrm{mg} / \mathrm{L})$, tigecycline $(0.06 \mathrm{mg} / \mathrm{L})$, and vancomycin $(0.5 \mathrm{mg} / \mathrm{L})$.

As one of the common pathogens of CAP, Haemophilus influenzae was relatively more susceptible to nemonoxacin than fluoroquinolones, evidenced by $\mathrm{MIC}_{90}(4 \mathrm{mg} / \mathrm{L})$ at least 2 -fold lower than ciprofloxacin $(16 \mathrm{mg} / \mathrm{L})$, levofloxacin $(8 \mathrm{mg} / \mathrm{L})$, and moxifloxacin $(>8 \mathrm{mg} / \mathrm{L})($ Table 1$){ }^{6}$

As for atypical pathogens, the in vitro activity of nemonoxacin $\left(\mathrm{MIC}_{90} 0.06 \mathrm{mg} / \mathrm{L}\right)$ against $C$. trachomatis was 2- to 4-fold more active than levofloxacin $\left(\mathrm{MIC}_{90} 0.25 \mathrm{mg} / \mathrm{L}\right)$ and doxycycline ( $\mathrm{MIC}_{90} 0.125 \mathrm{mg} / \mathrm{L}$ ), but 4-fold less active than azithromycin ( $\mathrm{MIC}_{90} 0.015 \mathrm{mg} / \mathrm{L}$ ). For C. pneumoniae, the activity of nemonoxacin $\left(\mathrm{MIC}_{90} 0.06 \mathrm{mg} / \mathrm{L}\right.$ ) was comparable to doxycycline ( $\mathrm{MIC}_{90} 0.125 \mathrm{mg} / \mathrm{L}$ ) and azithromycin $\left(\mathrm{MIC}_{90} 0.06 \mathrm{mg} / \mathrm{L}\right)$, but much better than levofloxacin $\left(\mathrm{MIC}_{90}\right.$ $0.5 \mathrm{mg} / \mathrm{L}$ ) (Table 1). ${ }^{7}$ Unfortunately, the data about the in vitro activity of nemonoxacin against Mycoplasma and Legionella pneumophila are lacking.

Nemonoxacin showed greater activity than fluoroquinolone comparators against the methicillin-susceptible Staphylococcus aureus (MSSA) strains. ${ }^{4}$ Its in vitro activity is slightly greater than fluoroquinolones against the methicillin-resistant Staphylococcus aureus (MRSA) (Table 1). Nemonoxacin remained highly active against community-acquired (CA)-MRSA, while relatively higher nemonoxacin MICs $(\geq 4 \mathrm{mg} / \mathrm{L}$ ) were noted among the hospital-acquired MRSA $(78 \% ; 77 / 99) .{ }^{4}$ According to the researches from Taiwan, nemonoxacin demonstrated activity superior to levofloxacin against CA-MRSA isolates, but similar to moxifloxacin and gemifloxacin. ${ }^{5}$ Nemonoxacin had slightly lower $\mathrm{MIC}_{90}(1 \mathrm{mg} / \mathrm{L})$ for CAMRSA than ceftobiprole, vancomycin, teicoplanin, and linezolid ( $2 \mathrm{mg} / \mathrm{L}$, respectively), but higher than daptomycin 
Table I In vitro antibacterial activities of nemonoxacin and comparators against bacteria

\begin{tabular}{|c|c|c|c|c|c|c|c|}
\hline \multirow{2}{*}{\multicolumn{4}{|c|}{ comparators against bacteria }} & \multirow{3}{*}{$\begin{array}{l}\text { Organism } \\
\text { (\# of isolates) }\end{array}$} & \multirow{3}{*}{ Quinolones } & \multirow{2}{*}{\multicolumn{2}{|c|}{ MIC (mg/L) }} \\
\hline & & & & & & & \\
\hline \multirow{2}{*}{$\begin{array}{l}\text { Organism } \\
\text { (\# of isolates) }\end{array}$} & \multirow[t]{2}{*}{ Quinolones } & \multicolumn{2}{|l|}{ MIC (mg/L) } & & & Range & $\mathrm{MIC}_{90}$ \\
\hline & & Range & $\mathrm{MIC}_{90}$ & Methicillin-resistant & Nemonoxacin & $0.015-64$ & 16 \\
\hline \multirow[t]{4}{*}{ S. pneumoniae (655) } & Nemonoxacin & $\leq 0.008-0.25$ & 0.015 & S. aureus (MRSA) (I27) & Ciprofloxacin & $0.25-16$ & 16 \\
\hline & Ciprofloxacin & $0.06-16$ & 2 & & Levofloxacin & $0.12-32$ & 32 \\
\hline & Levofloxacin & $0.06-32$ & I & & Moxifloxacin & $0.06-16$ & 16 \\
\hline & Moxifloxacin & $0.06-8$ & 0.25 & Ciprofloxacin-susceptible & Nemonoxacin & $\leq 0.03-0.06$ & $\leq 0.03$ \\
\hline Levofloxacin-susceptible & Nemonoxacin & $0.06-0.25$ & 0.12 & MRSA (50) & & & \\
\hline \multirow[t]{3}{*}{ S. pneumoniae (7I) } & Ciprofloxacin & $1-16$ & 4 & Ciprofloxacin-susceptible & Nemonoxacin & $\leq 0.008-0.06$ & 0.06 \\
\hline & Levofloxacin & $0.5-2$ & 2 & MRSA (44) & Ciprofloxacin & $\leq 0.06-1$ & 0.5 \\
\hline & Moxifloxacin & $0.06-0.5$ & 0.25 & & Levofloxacin & $\leq 0.06-0.25$ & 0.25 \\
\hline Levofloxacin-resistant & Nemonoxacin & $0.5-8$ & 2 & & Moxifloxacin & $\leq 0.015-0.12$ & 0.06 \\
\hline \multirow[t]{3}{*}{ S. pneumoniae (29) } & Ciprofloxacin & 8 to $>16$ & $>16$ & Ciprofloxacin-resistant & Nemonoxacin & $0.5-1$ & I \\
\hline & Levofloxacin & 8 to $>16$ & $>16$ & MRSA $(100)$ & & & \\
\hline & Moxifloxacin & 2 to $>8$ & 8 & Ciprofloxacin-resistant & Nemonoxacin & $0.06-4$ & 1 \\
\hline Penicillin-resistant & Nemonoxacin & $0.015-0.06$ & 0.03 & MRSA (47) & Ciprofloxacin & 2 to $>16$ & $>16$ \\
\hline \multirow[t]{3}{*}{ S. pneumoniae (32) } & Ciprofloxacin & $0.5-8$ & 2 & & Levofloxacin & 0.5 to $>16$ & 16 \\
\hline & Levofloxacin & $0.25-2$ & I & & Moxifloxacin & $0.12-8$ & 4 \\
\hline & Moxifloxacin & $0.06-0.25$ & 0.25 & Vancomycin-intermediate & Nemonoxacin & $0.03-8$ & 2 \\
\hline Levofloxacin- & Nemonoxacin & $0.12-4$ & I & MRSA (50) & & & \\
\hline nonsusceptible & & & & Daptomycin- & Nemonoxacin & $0.5-8$ & \\
\hline S. pneumoniae (30) & & & & nonsusceptible MRSA (5) & & & \\
\hline Invasive S. pneumoniae & Nemonoxacin & $\leq 0.03-1$ & 0.06 & Levofloxacin-susceptible & Nemonoxacin & $\leq 0.008-0.12$ & 0.06 \\
\hline$(150)$ & & & & MRSA (355) & Ciprofloxacin & $0.06-4$ & I \\
\hline \multirow[t]{4}{*}{ S. pyogenes $(30)$} & Nemonoxacin & $0.06-0.12$ & 0.12 & & Moxifloxacin & $0.06-0.25$ & 0.12 \\
\hline & Ciprofloxacin & $0.25-4$ & 4 & Levofloxacin- & Nemonoxacin & $0.25-64$ & 16 \\
\hline & Levofloxacin & $0.25-2$ & 2 & nonsusceptible MRSA & Ciprofloxacin & 16 & 16 \\
\hline & Moxifloxacin & $0.06-0.5$ & 0.5 & $(147)$ & Moxifloxacin & $1-16$ & 16 \\
\hline \multirow[t]{4}{*}{ S. agalactiae (30) } & Nemonoxacin & $0.12-2$ & 0.25 & Community-acquired & Nemonoxacin & $\leq 0.03-1$ & 0.06 \\
\hline & Ciprofloxacin & 1 to $>16$ & 2 & MRSA (I0I) & & & \\
\hline & Levofloxacin & 0.5 to $>16$ & 2 & Community-acquired & Nemonoxacin & $0.015-2$ & 0.5 \\
\hline & Moxifloxacin & 0.12 to $>8$ & 0.5 & MRSA (25) & Ciprofloxacin & $0.25-16$ & 16 \\
\hline \multirow[t]{4}{*}{ S. viridans (30) } & Nemonoxacin & $0.06-0.25$ & 0.25 & & Levofloxacin & $0.12-16$ & 8 \\
\hline & Ciprofloxacin & $0.25-4$ & 4 & & Moxifloxacin & $0.06-4$ & 2 \\
\hline & Levofloxacin & $0.25-2$ & 2 & Hospital-acquired MRSA & Nemonoxacin & $0.015-64$ & 16 \\
\hline & Moxifloxacin & $0.06-0.25$ & 0.25 & $(99)$ & Ciprofloxacin & $0.5-16$ & 16 \\
\hline \multirow[t]{4}{*}{ H. influenzae (30) } & Nemonoxacin & $\leq 0.008-8$ & 4 & & Levofloxacin & $0.25-32$ & 32 \\
\hline & Ciprofloxacin & $\leq 0.06-16$ & 16 & & Moxifloxacin & $0.06-16$ & 16 \\
\hline & Levofloxacin & $\leq 0.06-8$ & 8 & Coagulase-negative & Nemonoxacin & $0.03-8$ & 0.5 \\
\hline & Moxifloxacin & $\leq 0.015$ to $>8$ & $>8$ & Staphylococcus, methicillin- & Ciprofloxacin & 0.12 to $>16$ & $>16$ \\
\hline \multirow[t]{4}{*}{ C. pneumoniae (I0) } & Nemonoxacin & $0.03-0.125$ & 0.06 & resistant $(68)$ & Levofloxacin & 0.12 to $>16$ & 8 \\
\hline & Levofloxacin & 0.5 & 0.5 & & Moxifloxacin & 0.03 to $>8$ & 2 \\
\hline & Doxycycline & $0.06-0.125$ & 0.125 & Methicillin-susceptible & Nemonoxacin & $0.015-4$ & 0.5 \\
\hline & Azithromycin & $0.03-0.06$ & 0.06 & S. epidermidis (43) & Ciprofloxacin & $0.06-16$ & 16 \\
\hline \multirow[t]{4}{*}{ C. trachomatis (10) } & Nemonoxacin & $0.03-0.125$ & 0.06 & & Levofloxacin & $0.12-32$ & 32 \\
\hline & Levofloxacin & $0.125-0.5$ & 0.25 & & Moxifloxacin & $0.06-16$ & 4 \\
\hline & Doxycycline & $0.03-0.25$ & 0.125 & Methicillin-resistant & Nemonoxacin & $0.012-2$ & 2 \\
\hline & Azithromycin & $0.003-0.03$ & 0.015 & S. epidermidis (9) & Ciprofloxacin & $8-16$ & 16 \\
\hline Methicillin-susceptible & Nemonoxacin & $\leq 0.03-0.06$ & $\leq 0.03$ & & Levofloxacin & $4-32$ & 32 \\
\hline S. aureus $(100)$ & & & & & Moxifloxacin & $1-16$ & 16 \\
\hline Methicillin-susceptible & Nemonoxacin & $0.015-1$ & 0.12 & E. faecalis (3I) & Nemonoxacin & $0.12-4$ & 4 \\
\hline S. aureus (59) & & & & E. faecalis (8I) & Nemonoxacin & $0.03-128$ & I \\
\hline Methicillin-susceptible & Nemonoxacin & $\leq 0.008-32$ & 0.12 & & Ciprofloxacin & $0.25-16$ & 16 \\
\hline S. aureus (374) & Ciprofloxacin & $0.06-16$ & 4 & & Levofloxacin & $0.25-32$ & 32 \\
\hline & Levofloxacin & $0.06-32$ & I & & Moxifloxacin & $0.06-16$ & 16 \\
\hline & Moxifloxacin & $0.06-16$ & 0.25 & E. faecium (19) & Nemonoxacin & $0.5-8$ & 8 \\
\hline
\end{tabular}

Table I (Continued) 
Table I (Continued)

\begin{tabular}{|c|c|c|c|}
\hline \multirow{2}{*}{$\begin{array}{l}\text { Organism } \\
\text { (\# of isolates) }\end{array}$} & \multirow[t]{2}{*}{ Quinolones } & \multicolumn{2}{|l|}{ MIC (mg/L) } \\
\hline & & Range & $\mathrm{MIC}_{90}$ \\
\hline \multirow[t]{4}{*}{ E. faecium (38) } & Nemonoxacin & $0.06-128$ & 128 \\
\hline & Ciprofloxacin & $1-16$ & 16 \\
\hline & Levofloxacin & $\mathrm{I}-32$ & 32 \\
\hline & Moxifloxacin & $0.25-16$ & 16 \\
\hline $\begin{array}{l}\text { Vancomycin-susceptible } \\
\text { E. faecalis (50) }\end{array}$ & Nemonoxacin & $0.12-32$ & 0.5 \\
\hline \multicolumn{3}{|l|}{ E. faecalis (34) } & 4 \\
\hline \multicolumn{4}{|l|}{ E. faecium $(50)$} \\
\hline $\begin{array}{l}\text { Vancomycin-resistant } \\
\text { E. faecium (78) }\end{array}$ & Nemonoxacin & $0.06-16$ & 16 \\
\hline \multirow[t]{4}{*}{ E. coli (599) } & Nemonoxacin & $\leq 0.015$ to $\geq 512$ & 32 \\
\hline & Ciprofloxacin & $0.06-16$ & 16 \\
\hline & Levofloxacin & $0.06-32$ & 16 \\
\hline & Moxifloxacin & $0.06-16$ & 16 \\
\hline Ciprofloxacin-susceptible & Nemonoxacin & $0.015-4$ & 2 \\
\hline \multirow[t]{3}{*}{ E. coli $(37)$} & Ciprofloxacin & $\leq 0.06-1$ & I \\
\hline & Levofloxacin & $\leq 0.06-2$ & I \\
\hline & Moxifloxacin & $\leq 0.015-2$ & I \\
\hline Ciprofloxacin-resistant & Nemonoxacin & 2 to $>16$ & $>16$ \\
\hline \multirow[t]{3}{*}{ E. coli $(43)$} & Ciprofloxacin & 2 to $>16$ & $>16$ \\
\hline & Levofloxacin & I to $>16$ & $>16$ \\
\hline & Moxifloxacin & 2 to $>8$ & $>8$ \\
\hline K. pneumoniae (30) & Nemonoxacin & 0.25 to $>16$ & $>16$ \\
\hline \multirow[t]{4}{*}{ K. pneumoniae (199) } & Nemonoxacin & $\leq 0.015-128$ & 2 \\
\hline & Ciprofloxacin & $0.06-16$ & 0.5 \\
\hline & Levofloxacin & $0.06-32$ & I \\
\hline & Moxifloxacin & $0.06-16$ & I \\
\hline \multirow[t]{4}{*}{ K. oxytoca $(30)$} & Nemonoxacin & $0.06-1$ & 0.5 \\
\hline & Ciprofloxacin & $0.06-0.25$ & 0.06 \\
\hline & Levofloxacin & $0.06-0.25$ & 0.06 \\
\hline & Moxifloxacin & $0.06-0.25$ & 0.12 \\
\hline E. cloacae (30) & Nemonoxacin & 0.5 to $>16$ & $>16$ \\
\hline \multirow[t]{4}{*}{ E. cloacae (72) } & Nemonoxacin & $0.06-32$ & 0.5 \\
\hline & Ciprofloxacin & $0.06-16$ & 0.12 \\
\hline & Levofloxacin & $0.06-32$ & 0.25 \\
\hline & Moxifloxacin & $0.06-16$ & 0.25 \\
\hline P. mirabilis (30) & Nemonoxacin & 0.5 to $>16$ & $>16$ \\
\hline \multirow[t]{4}{*}{ P. mirabilis (33) } & Nemonoxacin & 0.25 to $\geq 512$ & 16 \\
\hline & Ciprofloxacin & $0.06-2$ & 2 \\
\hline & Levofloxacin & $0.06-32$ & 4 \\
\hline & Moxifloxacin & $0.12-16$ & 16 \\
\hline P. aeruginosa (30) & Nemonoxacin & I to $>16$ & $>16$ \\
\hline \multirow[t]{4}{*}{ P. aeruginosa (137) } & Nemonoxacin & $\leq 0.015$ to $\geq 512$ & 32 \\
\hline & Ciprofloxacin & $0.06-16$ & 8 \\
\hline & Levofloxacin & $0.06-32$ & 16 \\
\hline & Moxifloxacin & $0.06-16$ & 16 \\
\hline A. baumannii (30) & Nemonoxacin & 0.06 to $>16$ & $>16$ \\
\hline \multirow[t]{4}{*}{ A. baumannii (I5) } & Nemonoxacin & $0.12-16$ & I \\
\hline & Ciprofloxacin & $0.12-32$ & 4 \\
\hline & Levofloxacin & $0.06-16$ & 1 \\
\hline & Moxifloxacin & $0.06-4$ & 0.5 \\
\hline
\end{tabular}

(Continued)
Table I (Continued)

\begin{tabular}{llll}
\hline $\begin{array}{l}\text { Organism } \\
\text { (\# of isolates) }\end{array}$ & Quinolones & \multicolumn{2}{l}{ MIC (mg/L) } \\
\cline { 3 - 4 } & & Range & MIC $_{90}$ \\
\hline S. maltophilia (26) & Nemonoxacin & $0.25-64$ & 32 \\
& Ciprofloxacin & $0.06-16$ & 16 \\
& Levofloxacin & $0.06-16$ & 8 \\
& Moxifloxacin & $0.12-8$ & 4 \\
C. freundii (30) & Nemonoxacin & 0.12 to $>16$ & 4 \\
& Ciprofloxacin & $\leq 0.06$ to $>16$ & 2 \\
& Levofloxacin & $\leq 0.06$ to $>16$ & 2 \\
& Moxifloxacin & 0.12 to $>8$ & 8 \\
\hline
\end{tabular}

Note: Data from Adam HJ et al, Chen YH et al, Lauderdale TL et al, Chotikanatis K, Kohlhoff SA, Hammerschlag MR. ${ }^{4-7}$

Abbreviations: MIC, minimum inhibitory concentration; MRSA, methicillinresistant Staphylococcus aureus.

$(0.25 \mathrm{mg} / \mathrm{L})$, tigecycline $(0.5 \mathrm{mg} / \mathrm{L})$, and mupirocin $(0.25 \mathrm{mg} / \mathrm{L}){ }^{6,8}$ However, nemonoxacin had limited activities against vancomycin-intermediate MRSA (MIC $\geq 0.5 \mathrm{mg} / \mathrm{L}$ for $66 \%$ [33/50] isolates, $\mathrm{MIC}_{90}=2 \mathrm{mg} / \mathrm{L}$ ) and daptomycin nonsusceptible MRSA. ${ }^{5}$

Nemonoxacin had stronger inhibitory activity against both methicillin-susceptible S. epidermidis (MSSE) and methicillin-resistant $S$. epidermidis (MRSE) than ciprofloxacin, levofloxacin, and moxifloxacin., ${ }^{4,6}$ The $\mathrm{MIC}_{90}$ values of nemonoxacin against MSSE and MRSE were much lower than those of the fluoroquinolones. ${ }^{4}$ Similarly, Lauderdale et $\mathrm{al}^{6}$ reported much lower $\mathrm{MIC}_{90}$ of nemonoxacin against MRSE $(0.5 \mathrm{mg} / \mathrm{L})$ than ciprofloxacin $(>16 \mathrm{mg} / \mathrm{L})$, levofloxacin $(8 \mathrm{mg} / \mathrm{L})$, moxifloxacin $(2 \mathrm{mg} / \mathrm{L})$, ceftriaxone $(>8 \mathrm{mg} / \mathrm{L})$, linezolid (2 mg/L), and vancomycin $(2 \mathrm{mg} / \mathrm{L})^{6}$ (Table 1$)$.

Nemonoxacin also displayed greater activity than the fluoroquinolone comparators against E. faecalis. ${ }^{4-6}$ In the testing of 31 strains of $E$. faecalis, nemonoxacin $\mathrm{MIC}_{90}$ value was at least 2-fold lower than that of ciprofloxacin, levofloxacin, and moxifloxacin, and also lower than vancomycin. ${ }^{6}$ These results were consistent with the report from Canada. ${ }^{4}$ Nemonoxacin was not so active against $E$. faecium in vitro. Vancomycin-susceptible Enterococcus isolates were more sensitive to nemonoxacin than vancomycin-resistant strains (Table 1). ${ }^{5}$

Generally, nemonoxacin is not as active for gramnegative bacterial isolates as for gram-positive cocci. Its in vitro activity against Enterobacteriaceae isolates was similar or slightly inferior to the fluoroquinolones tested. ${ }^{4,6}$ Nemonoxacin showed high MIC $\left(\mathrm{MIC}_{90}>32 \mathrm{mg} / \mathrm{L}\right)$ and low susceptibility to ertapenem-nonsusceptible Enterobacteriaceae. ${ }^{9,10}$

As for the nonfermenters - such as Pseudomonas aeruginosa, Stenotrophomonas maltophilia, and 
Acinetobacter baumannii - nemonoxacin generally showed similar activity as other fluoroquinolone comparators. ${ }^{4,6}$ It was reported that nemonoxacin $\left(\mathrm{MIC}_{90} 0.25 \mathrm{mg} / \mathrm{L}\right)$ had 1- to 2-fold lower MIC against Helicobacter pylori than ciprofloxacin, levofloxacin, and moxifloxacin. ${ }^{11}$ In a research study from Taiwan, the activity of quinolones against clinical isolates of Clostridium difficile indicated that nemonoxacin was better than moxifloxacin, gemifloxacin, levofloxacin, and ciprofloxacin. ${ }^{12,13}$ The antimicrobial activity of nemonoxacin against other anaerobes was not reported yet. In the testing of clinical isolates of more than 20 Nocardia species, nemonoxacin showed the lowest $\mathrm{MIC}_{90}$ values $(0.5-8 \mathrm{mg} / \mathrm{L})$ among the tested quinolones. ${ }^{14,15}$ Nemonoxacin was less active against Mycobacterium tuberculosis than other quinolones, according to the study from Taiwan. ${ }^{16}$

\section{Resistance induction}

Three unrelated clinical isolates of $S$. pneumoniae were exposed to nemonoxacin to assess the potential of $S$. pneumoniae to develop resistance to nemonoxacin and to characterize the mutations in topoisomerase II and topoisomerase IV (target genes of fluoroquinolones) associated with nemonoxacin resistance. The MICs of nemonoxacin increased 2- to 8-fold over three cycles of selection (initial MIC 0.03-0.06 mg/L versus final MIC 0.06-0.5 mg/L). Importantly, no highly resistant isolates were induced. In contrast, the MIC of ciprofloxacin had a 64- to 128-fold increase over three selection cycles (initial MIC $0.5 \mathrm{mg} / \mathrm{L}$ versus final MIC 32-64 mg/L) and yielded highly resistant isolates. The final MICs were: $0.5-1 \mathrm{mg} / \mathrm{L}$ for nemonoxacin; $4 \mathrm{mg} / \mathrm{L}$ for moxifloxacin; 4-8 mg/L for gatifloxacin; and $16 \mathrm{mg} / \mathrm{L}$ for levofloxacin. ${ }^{17}$ After exposure to nemonoxacin, the identified mutations included Ser82Tyr in gyrA, Ser494Thr in $g y r B$, and Pro454Ser in parE. Nemonoxacin has a low propensity for selecting resistant pathogens compared to other fluoroquinolones, because the bacteria become resistant to nemonoxacin only when three different mutations occur in their quinolone resistance-determining region of the relevant genes. ${ }^{17}$

\section{In vivo antibacterial efficacy}

The in vivo efficacy of nemonoxacin was evaluated in mouse models of systemic and local (pulmonary and ascending urinary tract) infections using levofloxacin as a comparator. ${ }^{18}$ Used in the infection models were: MSSA (one strain); MRSA (one strain); levofloxacin- and methicillin-resistant S. capitis (one strain); penicillin-intermediate $S$. pneumoniae (PISP, one strain); penicillin-resistant S. pneumoniae (PRSP, two strains); vancomycin-resistant Enterococcus (VRE, two strains); and Escherichia coli (three strains) isolates. In a systemic infection model, nemonoxacin demonstrated higher therapeutic efficacy against MSSA, MRSA, methicillinresistant $S$. capitis, PISP, PRSP, and VRE infections with lower effective dose $(\mathrm{ED})_{50}$ and MIC values than levofloxacin $(P<0.01) .{ }^{18}$ Nemonoxacin also exhibited potent activity against $E$. coli, but the efficacy was lower than that of levofloxacin $(P<0.01)$ (Table 2$){ }^{18}$

Another study evaluated the in vivo activity of nemonoxacin in acute murine systemic infections induced by $S$. aureus, S. pneumoniae, E. coli, ciprofloxacin-resistant $S$. pneumoniae, and ciprofloxacin-resistant MRSA in comparison with

Table 2 In vivo efficacy of nemonoxacin and the reference compound in mouse systemic infection

\begin{tabular}{|c|c|c|c|c|c|c|}
\hline \multirow[t]{2}{*}{ Organism } & \multirow{2}{*}{$\begin{array}{l}\text { Challenge dose } \\
\text { (CFU/mouse) }\end{array}$} & \multicolumn{2}{|c|}{ Nemonoxacin } & \multicolumn{2}{|l|}{ Levofloxacin } & \multirow[t]{2}{*}{$P$-value } \\
\hline & & MIC (mg/L) & $E D_{50}(95 \% \mathrm{Cl})(\mathrm{mg} / \mathrm{kg})$ & MIC (mg/L) & $\mathrm{ED}_{50}(95 \% \mathrm{CI})(\mathrm{mg} / \mathrm{kg})$ & \\
\hline $\begin{array}{l}\text { S. aureus, ATCC } 29213 \\
\text { (MSSA) }\end{array}$ & $2.1 \times 10^{5}$ & 0.03 & $2.08(1.68-2.59)$ & 0.125 & $5.02(4.02-6.25)$ & $<0.01$ \\
\hline S. aureus 0705 (MRSA) & $2.5 \times 10^{5}$ & 0.03 & $2.59(2.24-2.98)$ & 0.25 & $8.45(7.60-9.41)$ & $<0.01$ \\
\hline $\begin{array}{l}\text { S. capitis } 0687 \text { (levofloxacin- } \\
\text { resistant MRSC) }\end{array}$ & $8.8 \times 10^{4}$ & 0.5 & $2.52(2.17-2.93)$ & 8 & $4.32(3.96-4.72)$ & $<0.01$ \\
\hline $\begin{array}{l}\text { S. pneumonia ATCC } 49619 \\
\text { (PISP) }\end{array}$ & $8.8 \times 10^{5}$ & 0.125 & $5.47(4.55-6.57)$ & 1 & 19.14 (16.98-21.57) & $<0.01$ \\
\hline S. pneumonia 05 I 8 (PRSP) & $2.2 \times 10^{5}$ & 0.25 & $3.68(3.11-4.36)$ & 2 & 19.82 (17.57-22.37) & $<0.01$ \\
\hline S. pneumonia 0613 (PRSP) & $1.8 \times 10^{5}$ & 0.25 & $5.28(4.21-6.62)$ & 2 & 22.01 (19.50-24.83) & $<0.01$ \\
\hline E. faecalis ATCC 29212 & $3.3 \times 10^{6}$ & 0.03 & $15.16(12.64-18.17)$ & 0.5 & $26.89(23.25-31.09)$ & $<0.01$ \\
\hline E. faecalis 404I (VRE) & $1.1 \times 10^{8}$ & 0.06 & $8.48(6.88-10.45)$ & I & 17.47 (|4.|8-2|.53) & $<0.01$ \\
\hline E. coli ATCC 25922 & $6.0 \times 10^{5}$ & 0.03 & $3.13(2.40-4.10)$ & 0.015 & $0.68(0.56-0.83)$ & $<0.01$ \\
\hline E. coli 0635 & $1.5 \times 10^{5}$ & 0.06 & $3.28(2.85-4.01)$ & 0.015 & $0.97(0.85-I .12)$ & $<0.01$ \\
\hline E. coli 0638 & $1.0 \times 10^{5}$ & 0.06 & $5.28(4.58-6.09)$ & 0.015 & $0.84(0.73-0.96)$ & $<0.01$ \\
\hline
\end{tabular}

Note: Data from Li et al. ${ }^{18}$

Abbreviations: $\mathrm{ED}_{50}, 50 \%$ effective dose; ATCC, American Type Culture Collection (ATCC), Manassas, VA, USA; CFU, colony-forming units; MIC, minimum inhibitory concentration; Cl, confidence interval; MSSA, methicillin-susceptible S. aureus; MRSA, methicillin-resistant S. aureus; MRSC, methicillin-resistant S. capitis; PISP, penicillinintermediate S. pneumoniae; PRSP, penicillin-resistant S. pneumoniae; VRE, vancomycin-resistant Enterococcus. 
ciprofloxacin, moxifloxacin, levofloxacin, gatifloxacin, and garenoxacin. The $\mathrm{PD}_{50}$ (the drug dose required to protect $50 \%$ of mice from death) values of nemonoxacin were lower than all the tested fluoroquinolones against ciprofloxacin-resistant MRSA, lower than ciprofloxacin, levofloxacin, moxifloxacin, and gatifloxacin against $S$. aureus, S. pneumoniae, and ciprofloxacin-resistant S. pneumoniae, but higher than all the fluoroquinolones against E. coli. ${ }^{19}$

In the mouse pulmonary infection model, nemonoxacin showed higher activity against PRSP (log colony-forming units [CFU]/g values: 2.93 versus 4.34 at $20 \mathrm{mg} / \mathrm{kg}$ dose level; 4.01 versus 4.76 at $10 \mathrm{mg} / \mathrm{kg}$ dose level and 4.81 versus 6.29 at $5 \mathrm{mg} / \mathrm{kg}$ dose level; all $P>0.05$ ) compared with levofloxacin at the same doses. However, it demonstrated lower activity toward Klebsiella pneumoniae (log CFU/g values, 4.49 versus 3.02 at $20 \mathrm{mg} / \mathrm{kg}$ dose level and 5.88 versus 3.33 at $10 \mathrm{mg} / \mathrm{kg}$ dose level; all $P<0.05) .{ }^{18}$ Another study reported the efficacy of nemonoxacin and moxifloxacin in a mouse $S$. pneumoniae pulmonary infection model and found nemonoxacin more effective than moxifloxacin in reducing $S$. pneumoniae colonies and protecting mice from death. ${ }^{20}$

In the ascending urinary tract infection model, nemonoxacin and levofloxacin showed therapeutic efficacy associated with significantly decreased kidney colony counts in comparison with control group, even though the efficacy of nemonoxacin was lower than that of levofloxacin, based on $\log \mathrm{CFU} / \mathrm{g}$ values of 4.60 versus 3.47 at the $4 \mathrm{mg} / \mathrm{kg}$ dose level $(P<0.05) .{ }^{18}$

\section{Clinical studies}

Phase I: PK

The PK profile and tolerability of nemonoxacin were investigated in the USA $(25,50,100,125,250,500,1,000$, and 1,500 mg single dose), ${ }^{21}$ the People's Republic of China $(125,250,500,750$, and 1,000 mg single dose and multiple doses for 10 days), ${ }^{22,23}$ and Taiwan (75, 250, 500, 750, and $1,000 \mathrm{mg}$ once a day for 10 days) (Tables 3 and 4). ${ }^{24}$

In the single-dose trials in healthy volunteers, $\mathrm{C}_{\max }$ of nemonoxacin was dose proportional over the dose range from $500-1,500 \mathrm{mg} .{ }^{21,23}$ Nemonoxacin rapidly reached its $\mathrm{C}_{\max }$ within 1-2 hours after a single oral administration (dose range of $25,50,125,250,500,750,1,000$, and $1,500 \mathrm{mg}$ ) in the fasting state. ${ }^{21-24}$ Following a single dose of $500 \mathrm{mg}$, the average $\mathrm{C}_{\max }$ was $3.41-5.91 \mathrm{mg} / \mathrm{L}$ (Table 3). ${ }^{21,23}$

The mean plasma protein binding of nemonoxacin was approximately $16 \%$, which was lower than that of the existing fluoroquinolones, indicating its higher percentage of tissue penetration and lower potential for protein binding-based drug-drug interactions. ${ }^{21,23}$ The apparent $\mathrm{V}_{\mathrm{zF}}$ of nemonoxacin (3.81-4.25 L/kg after a single dose of $500 \mathrm{mg}$ ) exceeded the total body volume in the study population, suggesting an extensive tissue distribution pattern, which was similar to the existing fluoroquinolones. ${ }^{21,23}$

Nemonoxacin had a long elimination half-life of 10-15 hours, corresponding to the increased doses. ${ }^{21-24}$ Approximately $60 \%-75 \%$ of the administered dose

Table 3 Mean (standard deviation) PK parameters of nemonoxacin after oral single-dose administration

\begin{tabular}{|c|c|c|c|c|c|c|c|c|c|}
\hline Dose (mg) & $\begin{array}{l}C_{\max } \\
(\mathrm{mg} / \mathrm{L})\end{array}$ & $\begin{array}{l}T_{\max } \\
\text { (h) }\end{array}$ & $\begin{array}{l}\text { AUC }_{72} \\
(\mathrm{mg} \cdot \mathrm{h} / \mathrm{L})\end{array}$ & $\begin{array}{l}\text { AUC }_{0-\infty} \\
(\mathrm{mg} \cdot \mathrm{h} / \mathrm{L})\end{array}$ & $\begin{array}{l}T_{1 / 2} \\
\text { (h) }\end{array}$ & $\begin{array}{l}\mathrm{CL}_{\mathrm{T}} \\
(\mathrm{L} / \mathrm{h} / \mathrm{kg})\end{array}$ & $\begin{array}{l}\mathrm{CL}_{\mathrm{R}} \\
(\mathrm{L} / \mathrm{h} / \mathrm{kg})\end{array}$ & $V_{d}(L / k g)$ & $\begin{array}{l}\mathrm{Ae}_{(72 \mathrm{~h})} \\
(\%)\end{array}$ \\
\hline \multicolumn{10}{|l|}{ Guo et $\mathrm{al}^{23, \mathrm{a}}$} \\
\hline $250(n=12)$ & $\begin{array}{l}3.24 \\
(0.67)\end{array}$ & $\begin{array}{l}1.04 \\
(0.69)\end{array}$ & $\begin{array}{l}21.40 \\
(3.35)\end{array}$ & $\begin{array}{l}21.52 \\
(3.36)\end{array}$ & $\begin{array}{l}10.73 \\
(2.71)\end{array}$ & $\begin{array}{l}0.20 \\
(0.035)\end{array}$ & $\begin{array}{l}0.14 \\
(0.034)\end{array}$ & $\begin{array}{l}3.08 \\
(1.09)\end{array}$ & $\begin{array}{l}70.28 \\
(7.55)\end{array}$ \\
\hline $500(n=11)$ & $\begin{array}{l}5.91 \\
(1.35)\end{array}$ & $\begin{array}{l}1.14 \\
(0.64)\end{array}$ & $\begin{array}{l}42.17 \\
(5.84)\end{array}$ & $\begin{array}{l}42.41 \\
(5.83)\end{array}$ & $\begin{array}{l}12.83 \\
(3.72)\end{array}$ & $\begin{array}{l}0.20 \\
(0.033)\end{array}$ & $\begin{array}{l}0.14 \\
(0.037)\end{array}$ & $\begin{array}{l}3.81 \\
(1.43)\end{array}$ & $\begin{array}{l}69.12 \\
(10.80)\end{array}$ \\
\hline $750(n=12)$ & $\begin{array}{l}8.20 \\
(1.37)\end{array}$ & $\begin{array}{l}1.64 \\
(0.60)\end{array}$ & $\begin{array}{l}64.75 \\
(6.24)\end{array}$ & $\begin{array}{l}65.04 \\
(6.23)\end{array}$ & $\begin{array}{l}10.92 \\
(3.78)\end{array}$ & $\begin{array}{l}0.19 \\
(0.033)\end{array}$ & $\begin{array}{l}0.13 \\
(0.027)\end{array}$ & $\begin{array}{l}3.00 \\
(1.02)\end{array}$ & $\begin{array}{l}66.00 \\
(8.66)\end{array}$ \\
\hline $\begin{array}{l}500 \text { after meal } \\
(n=I I)\end{array}$ & $\begin{array}{l}3.90 \\
(0.87)\end{array}$ & $\begin{array}{l}3.64 \\
(1.12)\end{array}$ & $\begin{array}{l}34.24 \\
(4.60)\end{array}$ & $\begin{array}{l}34.53 \\
(4.58)\end{array}$ & $\begin{array}{l}14.99 \\
(4.96)\end{array}$ & $\begin{array}{l}0.25 \\
(0.045)\end{array}$ & $\begin{array}{l}0.14 \\
(0.030)\end{array}$ & $\begin{array}{l}5.35 \\
(2.00)\end{array}$ & $\begin{array}{l}54.25 \\
(4.58)\end{array}$ \\
\hline Lin et $\mathrm{al}^{21, \mathrm{~b}}$ & & & & & & & & & \\
\hline $250(n=8)$ & $\begin{array}{l}2.40 \\
(0.66)\end{array}$ & $\begin{array}{l}0.92 \\
(0.20)\end{array}$ & & $\begin{array}{l}15.45 \\
(3.94)\end{array}$ & $\begin{array}{l}10.86 \\
(3.91)\end{array}$ & $\begin{array}{l}0.24 \\
(0.056)\end{array}$ & $\begin{array}{l}0.11 \\
(0.055)\end{array}$ & $\begin{array}{l}3.58 \\
(0.89)\end{array}$ & $\begin{array}{l}44.85 \\
(17.48)\end{array}$ \\
\hline $500(n=8)$ & $\begin{array}{l}3.41 \\
(0.58)\end{array}$ & $\begin{array}{l}2.0 \\
(0.87)\end{array}$ & & $\begin{array}{l}32.36 \\
(3.01)\end{array}$ & $\begin{array}{l}14.75 \\
(3.06)\end{array}$ & $\begin{array}{l}0.20 \\
(0.024)\end{array}$ & $\begin{array}{l}0.068 \\
(0.021)\end{array}$ & $\begin{array}{l}4.25 \\
(1.31)\end{array}$ & $\begin{array}{l}34.15 \\
(9.05)\end{array}$ \\
\hline $1,000(n=8)$ & $\begin{array}{l}7.22 \\
(0.88)\end{array}$ & $\begin{array}{l}1.67 \\
(0.26)\end{array}$ & & $\begin{array}{l}63.31 \\
(10.01)\end{array}$ & $\begin{array}{l}16.41 \\
(2.54)\end{array}$ & $\begin{array}{l}0.22 \\
(0.025)\end{array}$ & $\begin{array}{l}0.084 \\
(0.03 I)\end{array}$ & $\begin{array}{l}5.28 \\
(0.78)\end{array}$ & $\begin{array}{l}36.54 \\
(12.03)\end{array}$ \\
\hline
\end{tabular}

Notes: Copyright (C) 2010. Amended with permission from American Society for Microbiology. Lin L, Chang LW, Tsai CY, et al. Dose Escalation Study of the Safety, Tolerability, and Pharmacokinetics of Nemonoxacin (TG-873870), a Novel Potent Broad-Spectrum Nonfluorinated Quinolone, in Healthy Volunteers. Antimicrob Agents Chemother. 2010;54(I):405-4I0. doi: 10.1 I28/AAC.00682-09.21 Copyright @ 2012. Adapted with kind permission from Springer Science and Business Media. Guo B, Wu X, Zhang $Y$, et al. Safety and clinical pharmacokinetics of nemonoxacin, a novel non-fluorinated quinolone, in healthy Chinese volunteers following single and multiple oral doses. Clin Drug Investig. 20I2;32(7):475-486. ${ }^{23}$ aChinese population; ${ }^{\mathrm{b}}$ multiracial group including Hispanic (7I\%), African American (I3\%), and Caucasian (9\%).

Abbreviations: $\mathrm{PK}$, pharmacokinetics; $\mathrm{C}_{\max }$, maximum concentration; $\mathrm{T}_{\max }$, time that $\mathrm{C}_{\max }$ occurs; $\mathrm{AUC}$, area under concentration-time curve; $\mathrm{T}_{1 / 2}$, elimination half-life; $\mathrm{CL}_{\mathrm{T}}$, total clearance; $\mathrm{CL}_{\mathrm{R}}$, renal clearance; $\mathrm{V}_{\mathrm{d}}$, apparent volume of distribution; $\mathrm{Ae}_{(72 h)}$, percentage of the administered dose recovered in urine over 72 hours. 
Table 4 Mean (standard deviation) PK parameters of nemonoxacin following single oral administration and once-daily oral administration for 10 days

\begin{tabular}{|c|c|c|c|c|c|c|}
\hline $\begin{array}{l}\text { Dose }(\mathrm{mg}) \text { group } \\
\text { and time }\end{array}$ & $C_{\max }(\mathrm{mg} / \mathrm{L})$ & $\mathbf{T}_{\max }(\mathbf{h})$ & $\mathrm{AUC}_{24}(\mathrm{mg} \cdot \mathrm{h} / \mathrm{L})$ & $A e_{(24 h)}(\%)$ & $C L_{R}(L / h / k g)$ & $\begin{array}{l}\text { Accumulation } \\
\text { index }\end{array}$ \\
\hline \multicolumn{7}{|l|}{ Guo et $\mathrm{al}^{23, \mathrm{c}}$} \\
\hline \multicolumn{7}{|l|}{$500(n=12)$} \\
\hline Day I & $6.46(1.29)$ & $1.42(0.90)$ & 43.93 (8.97) & $54.8 \mathrm{I}(\mathrm{II} .6 \mathrm{I})$ & $0.11(0.026)$ & NA \\
\hline Day 10 & 7.02 (1.77) & $1.25(0.45)$ & $46.92(12.15)$ & $50.81(16.07)$ & $0.10(0.042)$ & $1.09(0.05)$ \\
\hline \multicolumn{7}{|l|}{$750(n=12)$} \\
\hline Day I & $9.38(2.70)$ & $1.92(1.06)$ & $63.28(10.52)$ & $56.24(6.11)$ & $0.12(0.024)$ & NA \\
\hline Day 10 & $9.13(1.55)$ & $\mathrm{I} .46(0.8 \mathrm{I})$ & $65.75(9.06)$ & $65.38(6.81)$ & $0.13(0.024)$ & $1.10(0.05)$ \\
\hline \multicolumn{7}{|l|}{ Chung et $\mathrm{al}^{24, \mathrm{~d}}$} \\
\hline \multicolumn{7}{|l|}{$500(n=8)$} \\
\hline Day I & $5.12(1.04)$ & $1.00(0.5-1.5)^{\mathrm{a}}$ & 31.60 (4.33) & $42.2(13.1)$ & $6.92(3.02)^{b}$ & \\
\hline Day 10 & $5.56(1.39)$ & I.3। $(1.0-2.0)^{\mathrm{a}}$ & $38.60(7.37)$ & $57.8(9.6)$ & $7.85(2.8 \mathrm{I})^{\mathrm{b}}$ & \\
\hline \multicolumn{7}{|l|}{$750(n=8)$} \\
\hline Day I & $5.75(1.18)$ & $1.50(1.0-2.0)^{\mathrm{a}}$ & 46.06 (9.28) & 47.1 (10.9) & $7.96(2.32)^{b}$ & \\
\hline Day 10 & $6.82(I .8 I)$ & $1.51(1.0-2.0)^{\mathrm{a}}$ & $58.43(14.32)$ & $41.8(10.3)$ & $5.63(1.74)^{b}$ & \\
\hline \multicolumn{7}{|l|}{$\mathrm{I}, 000(\mathrm{n}=8)$} \\
\hline Day I & $7.75(2.15)$ & $2.00(1.0-4.0)^{\mathrm{a}}$ & $59.65(12.46)$ & $47.9(8.7)$ & $7.48(1.80)^{b}$ & \\
\hline Day 10 & $8.20(2.03)$ & $2.07(1.5-4.0)^{\mathrm{a}}$ & 74.84 (I4.27) & $48.6(13.7)$ & $6.87(2.63)^{\mathrm{b}}$ & \\
\hline
\end{tabular}

Notes: Copyright (C) 20I2. Adapted with kind permission from Springer Science and Business Media. Guo B, Wu X, Zhang Y, et al. Safety and clinical pharmacokinetics of nemonoxacin, a novel non-fluorinated quinolone, in healthy Chinese volunteers following single and multiple oral doses. Clin Drug Investig. 20I2;32(7):475-486. ${ }^{23}$ Copyright $(\subset$ 2010. Adapted with permission from American Society for Microbiology. Chung DT, Tsai CY, Chen SJ, et al. Multiple-dose safety, tolerability, and pharmacokinetics of oral nemonoxacin (TG-873870) in healthy volunteers. Antimicrob Agents Chemother. 20 I 0;54(I):4I I-4I 7. doi: 10.1 I 28/AAC.00683-09. . $^{24} \mathrm{~T}_{\text {max }}$ is represented by means (range); ${ }^{\mathrm{b}} \mathrm{CL}_{\mathrm{R}}$ result is expressed as $\mathrm{L} / \mathrm{h}$; ' Chinese population; ${ }^{\mathrm{d}}$ multiracial group, including African American (56.6\%), Caucasian (28.3\%), Hispanic (13\%), and Asian (2.2\%).

Abbreviations: PK, pharmacokinetic; $C_{\max }$, maximum concentration; $T_{\max }$, time that $C_{\max }$ occurs; $A U C$, area under concentration-time curve; $A e_{(24 h)}$, percentage of the administered dose recovered in urine over 24 hours; $\mathrm{CL}_{\mathrm{R}}$, renal clearance; $\mathrm{NA}$, not available.

was excreted in unchanged form via the kidneys over 24-72 hours. ${ }^{21-23}$ The average renal clearance was $140 \mathrm{~mL} / \mathrm{h} / \mathrm{kg}$ in Chinese volunteers, following a single dose of $500 \mathrm{mg}^{23}$ and $68 \mathrm{~mL} / \mathrm{h} / \mathrm{kg}$ in the multiracial group after an administration of the same dose. ${ }^{21}$

Food had a significant effect on the rate and extent of nemonoxacin absorption $(P<0.001)$, increasing the time to reach $\mathrm{C}_{\text {max }}$ from 1.14-3.64 hours and reducing $\mathrm{C}_{\text {max }}$ and area under concentration-time curve (AUC) by $34 \%$ and $18 \%$, respectively. Sex effect was not found on the PK parameters of nemonoxacin. The excretion rates in the fed state were $20 \%$ lower than those in the fasting state. ${ }^{23}$

The multiple-dose PK data suggested little drug accumulation in healthy subjects, evidenced by the plasma concentration which was similar on day 1 and day 10 after administration. ${ }^{23,24}$ The theoretical accumulation ratios (mean \pm standard deviation) of the $\mathrm{C}_{\max }$ and $\mathrm{AUC}_{0-24}$ were comparable on day 1 and day $10(1.21 \pm 0.06$ and $1.11 \pm 0.08$, respectively). A steady state was reached after 3 days. The mean $T_{1 / 2}$ of nemonoxacin was calculated as 19.65 hours in steady state following multiple doses (Table 4). When administered at a dose of $\geq 500 \mathrm{mg}$, the plasma concentration of free nemonoxacin maintained above $0.5 \mathrm{mg} / \mathrm{L}$ for the full 24-hour dosing interval, which was higher than the $\mathrm{MIC}_{90}$ values for a wide spectrum of gram-positive pathogens. ${ }^{24}$
In preclinical studies, it has been found that nemonoxacin neither induces nor inhibits human hepatic cytochrome P450 3A4 (CYP3A4) activity. ${ }^{25}$ Metabolism studies completed to date indicate that no metabolite or a minor metabolite $(<5 \%)$ of nemonoxacin was formed. ${ }^{25}$

\section{PK/PD profile}

Generally, in the PK/PD studies of fluoroquinolones against $S$. pneumonia, $\mathrm{AUC} / \mathrm{MIC}_{90}$ ratio $\geq 25$ to approximately 63 or $\mathrm{C}_{\max }$ ' $\mathrm{MIC}_{90}$ ratio of $\geq 8$ to approximately 10 is required to predict clinical and microbiological success and to limit the development of bacterial resistance. ${ }^{26} \mathrm{The}_{\mathrm{MIC}}$ of nemonoxacin for $S$. pneumoniae and $S$. aureus isolates was $\leq 0.125 \mathrm{mg} / \mathrm{L} .{ }^{4}$ Following administration of $500 \mathrm{mg}$ nemonoxacin, the free $\mathrm{AUC} / \mathrm{MIC}_{90}$ ratio and free $\mathrm{C}_{\max } / \mathrm{MIC}$ ratio were greater than 227 and 24, respectively. At a dose of $750 \mathrm{mg}$, nemonoxacin demonstrated excellent (free AUC/MIC ${ }_{90}>100$ ) $\mathrm{PK} / \mathrm{PD}$ profile against: MSSA ( $\geq 1533$ ); CA-MRSA (766.7); ciprofloxacin-susceptible MRSA ( $\geq 1533$ ); S. pneumoiae, including PRSP (>393); moderate (free AUC/MIC $\left.{ }_{90} 30-100\right) \mathrm{PK} / \mathrm{PD}$ profile against $S$. epidermidis (98); vancomycin-susceptible Enterococcus (92); ciprofloxacin-resistant MRSA (46); and unfavorable (free $\mathrm{AUC} / \mathrm{MIC}_{90}<30$ ) $\mathrm{PK} / \mathrm{PD}$ profile against vancomycin-intermediate $S$. aureus (VISA) and other entero$\operatorname{cocci}(<11.5))^{4,5,21}$ 
However, a study using an in vitro $S$. pneumoniae infection model (two-compartment model without absorption delay) indicated a dual-killing pattern of nemonoxacin. It is mainly concentration-dependent when the MIC is low. The better PK/ PD index should be the area under the concentration-time curve for the free, unbound fraction of the drug divided by the MIC ( $\mathrm{fAUC}_{0-24} / \mathrm{MIC}$ ). When the MIC is high, the time dependency is important. The valid PK/PD index should be the cumulative percentage of a 24-hour period during which the drug concentration exceeds the MIC under steady-state PK conditions $(\% \mathrm{fT}>\mathrm{MIC}){ }^{27}$

\section{Tolerability}

The Phase I studies of oral nemonoxacin with healthy volunteers showed good tolerance and safety. In the singledose study, nemonoxacin was well tolerated up to the maximum dose of 1,500 $\mathrm{mg}$. The most frequent adverse events (AE) were contact dermatitis $(12 \%)$, pruritus $(12 \%)$, and erythema (10\%). ${ }^{21}$ No QT interval prolongation, glucose homeostasis dysregulation, or hepatotoxicity was noted. ${ }^{21,23}$ In the multiple-dose study, the most common AEs were decreased WBC (29.2\%), rash (25\%), increased alanine aminotransferase (20.8\%), nausea (16.7\%), and elevated aspartate aminotransferase $(16.7 \%) .{ }^{23}$ None of the corrected QT values were more than $500 \mathrm{~ms}$, nor was the corrected QT values difference more than $60 \mathrm{~ms}$ - except in one subject from the $750 \mathrm{mg}$ multiple-dose study. ${ }^{23}$

\section{Phase II clinical trials}

Most CAP pathogens, including S. pneumoniae, H. influenzae, K. pneumoniae, and atypical pathogens ${ }^{28,29}$ were proved sensitive to nemonoxacin in the previous preclinical studies as mentioned in this review.

A randomized, double-blind, multicenter Phase II study compared the efficacy and safety of nemonoxacin $(500 \mathrm{mg}$ or $750 \mathrm{mg}$ once daily for 7 days) with levofloxacin (500 mg once daily for 7 days) in 265 adult patients with mild-to-moderate $\mathrm{CAP}^{30}{ }^{30}$ There were 86 patients in the $500 \mathrm{mg}$ nemonoxacin group, 89 in the $750 \mathrm{mg}$ nemonoxacin group, and 90 in the $500 \mathrm{mg}$ levofloxacin group. The primary endpoint was clinical cure rate at the test-of-cure visit. The bacteriological success rate at the test-of-cure visit was assessed in subjects with a baseline pathogen.

The clinical cure rate for $750 \mathrm{mg}$ nemonoxacin, $500 \mathrm{mg}$ nemonoxacin, and $500 \mathrm{mg}$ levofloxacin was $89.9 \%, 87.0 \%$, and $91.1 \%$, respectively, in the evaluable intent-to-treat (ITT) population; $91.7 \%, 87.7 \%$, and $90.3 \%$, respectively, in the evaluable per-protocol (PP) population. The 95\% confidence interval for the efficacy difference between $750 \mathrm{mg}$ nemonoxacin and $500 \mathrm{mg}$ levofloxacin was $-10.4 \%$ to $7.9 \%$ in the evaluable ITT population and $-8.0 \%$ to $10.8 \%$ in the evaluable PP population. These results indicate that nemonoxacin $750 \mathrm{mg}$ is noninferior to levofloxacin $500 \mathrm{mg}$. Similarly, noninferiority was demonstrated between nemonoxacin $500 \mathrm{mg}$ and levofloxacin at the same dose level with the $95 \%$ confidence interval of $-13.9 \%$ to $5.7 \%$ in the evaluable ITT population and $-12.8 \%$ to $7.6 \%$ in the evaluable PP population.

Overall bacteriological success rate was high for all treatment groups in the bacteriologically evaluable ITT population: $90.2 \%$ in the $750 \mathrm{mg}$ nemonoxacin group; $84.8 \%$ in the $500 \mathrm{mg}$ nemonoxacin group; and $92.0 \%$ in levofloxacin group. The bacteriological success rate for $S$. pneumoniae was $100 \%(5 / 5)$ in the $750 \mathrm{mg}$ nemonoxacin group, $75 \%$ $(3 / 4)$ in the $500 \mathrm{mg}$ nemonoxacin group, and $100 \%(5 / 5)$ in the $500 \mathrm{mg}$ levofloxacin group. For $H$. influenzae, the rate was $83 \%(5 / 6), 100 \%(4 / 4)$ and $100 \%(7 / 7)$, respectively. The most common atypical pathogen identified serologically was Mycoplasma pneumoniae. The bacteriological success rate was $89.7 \%$ (26/29) in $750 \mathrm{mg}$ nemonoxacin group, 80.6\% $(25 / 31)$ in $500 \mathrm{mg}$ nemonoxacin group, and 93.9\% (31/33) in levofloxacin group. For C. pneumoniae, the rate was $100 \%$ $(8 / 8), 100 \%(8 / 8)$, and $85.7 \%(6 / 7)$ in the three groups, respectively. The bacteriological success rate was $100 \%$ for L. pneumophila in all the three groups. For baseline pathogens, the nemonoxacin MIC range was 0.06 to $0.12 \mathrm{mg} / \mathrm{L}$ for $S$. pneumoniae $(\mathrm{n}=14)$, which is much lower than the MIC range of levofloxacin $(0.5-1 \mathrm{mg} / \mathrm{L})$. The MIC range of nemonoxacin for $S$. aureus $(\mathrm{n}=4)$ was also lower than that of levofloxacin (0.03-0.06 mg/L versus $0.12-0.5 \mathrm{mg} / \mathrm{L}$ ).

All the three treatments were well-tolerated without reports of drug-related serious AE. No clinically significant difference in drug-related AEs was noted among the three groups. The common AEs in nemonoxacin group occurred in the gastrointestinal $(17.4 \%$ in $750 \mathrm{mg}$ nemonoxacin group, $11.2 \%$ in $500 \mathrm{mg}$ nemonoxacin group, and $8.9 \%$ in levofloxacin group) and nervous system $(9.3 \%$ in $750 \mathrm{mg}$ nemonoxacin group, $6.7 \%$ in $500 \mathrm{mg}$ nemonoxacin group, and $4.4 \%$ in levofloxacin group), including diarrhea and dizziness. Nevertheless, no difference was found across the treatment groups in the incidence of drug related treatment emergent AEs (31.4\% in $750 \mathrm{mg}$ nemonoxacin group, 30.3\% in $500 \mathrm{mg}$ nemonoxacin group, and $30.0 \%$ in levofloxacin group) (Table 5). Nemonoxacin demonstrated a consistently favorable safety profile, as observed in the previous evaluations. In summary, nemonoxacin $500 \mathrm{mg}$ or $750 \mathrm{mg}$ once daily is as effective and well-tolerated as levofloxacin 
Table 5 Number and frequency of subjects with drug-related TEAE $(>2 \%)$ in the phase II clinical trial of nemonoxacin for treating community-acquired pneumonia

\begin{tabular}{|c|c|c|c|}
\hline \multirow[t]{2}{*}{ Adverse event } & \multicolumn{3}{|l|}{ Number (\%) of subjects } \\
\hline & Nemonoxacin 500 mg $(n=89)$ & Nemonoxacin 750 mg $(n=86)$ & Levofloxacin $500 \mathrm{mg}(\mathrm{n}=90)$ \\
\hline Subjects with any drug-related TEAE & $27(30.3)$ & $27(31.4)$ & $27(30.0)$ \\
\hline Neutropenia & $8(9.0)$ & $8(9.3)$ & $10(11.1)$ \\
\hline Dizziness & $4(4.5)$ & $3(3.5)$ & $2(2.2)$ \\
\hline Nausea & $\mathrm{I}(\mathrm{I} . \mathrm{I})$ & $5(5.8)$ & $3(3.3)$ \\
\hline Diarrhea & $5(5.6)$ & $\mathrm{I}(\mathrm{I} .2)$ & $\mathrm{I}(\mathrm{I} . \mathrm{I})$ \\
\hline Thrombocythemia & $2(2.2)$ & $4(4.7)$ & $\mathrm{I}(\mathrm{I} . \mathrm{I})$ \\
\hline ECG QTc interval prolonged & 0 & $2(2.3)$ & $3(3.3)$ \\
\hline Blood amylase increased & $\mathrm{I}(\mathrm{I} . \mathrm{I})$ & $\mathrm{I}(\mathrm{I} .2)$ & $2(2.2)$ \\
\hline Headache & $2(2.2)$ & $\mathrm{I}(\mathrm{I} .2)$ & 0 \\
\hline ALT increased & $2(2.2)$ & 0 & $\mathrm{I}(\mathrm{I} . \mathrm{I})$ \\
\hline AST increased & $2(2.2)$ & 0 & 0 \\
\hline
\end{tabular}

Note: Antimicrob Agents Chemother, 2010;54(10):4098-4106, doi: 10.1 I28/AAC.00295-10, amended with permission from American Society for Microbiology. ${ }^{30}$ Abbreviations: TEAE, treatment emergent adverse event; ECG, electrocardiogram; QTc, QT interval corrected; ALT, alanine aminotransferase; AST, aspartate aminotransferase.

$500 \mathrm{mg}$ once daily over a 7-day course for the treatment of adults with $\mathrm{CAP}^{30}$

In previous in vitro and animal infection models, nemonoxacin showed good activity against MSSA and MRSA. Open-label, noncomparative Phase II trials in patients with mild-to-moderate diabetic foot infection were conducted in US, South Africa, Thailand, and Taiwan. Patients were treated with nemonoxacin $750 \mathrm{mg}$ once daily for 1 or 2 weeks. Four of the 40 enrolled patients were MRSA-infected, including one patient with quinolone-resistant MRSA, who were successfully treated based on clinical evaluation (http://www. taigenbiotech.com/NewsDetail). Further clinical studies are warranted, based on this promising result. ${ }^{31}$

Five additional Phase II clinical trials on nemonoxacin are registered on the Internet database (clinicaltrials.gov). Three Phase II oral nemonoxacin studies on pneumonia had been completed, and one Phase II trial of intravenous infusion on pneumonia is still recruiting. The remaining one of oral nemonoxacin on diabetic foot infections was remarked with "completed," but no reports had been published.

\section{Conclusion}

Nemonoxacin, a nonfluorinated quinolone, has broadspectrum antibacterial activity covering most gram-positive cocci, atypical pathogens, and most gram-negative bacteria. It is especially more active than the commonly used fluoroquinolones against some typical antibiotic-resistant grampositive cocci, such as PRSP and MRSA.

Nemonoxacin demonstrated excellent efficacy in fighting systemic and local infections in murine models. The sound PK profile of nemonoxacin is characteristic of short time to reach $\mathrm{C}_{\max }$, long $\mathrm{T}_{1 / 2}$, low plasma protein-binding rate, and large apparent $\mathrm{V}_{\mathrm{z} / \mathrm{F}}$ similar to the fluoroquinolones. Its superior PK/PD features can predict sound clinical efficacy, which is reflected in clinical trial in CAP patients. Oral administration of nemonoxacin at a dose of $500 \mathrm{mg}$ and $750 \mathrm{mg}$ once daily for 7 days is proved as effective and safe as levofloxacin $500 \mathrm{mg}$ once a day in the patients with CAP. The upcoming results from the ongoing clinical trials will further prove the clinical value of oral and intravenous nemonoxacin in treating CAP and other relevant infections.

\section{Disclosure}

The authors report no conflicts of interest in this work. Some clinical trials of nemonoxacin cited in this review were conducted in Huashan Hospital, where authors Xiaohua Qin and Haihui Huang both work. However, neither of the authors were involved in these studies nor did they receive any financial support or other form of assistance from the company. The authors retained full editorial control over the content of the manuscript and received no compensation from any party for their work.

\section{References}

1. Rice LB. Mechanisms of resistance and clinical relevance of resistance to $\beta$-lactams, glycopeptides, and fluoroquinolones. Mayo Clin Proc. 2012;2(87):198-208.

2. Karpiuk I, Tyski S. Looking for the new preparations for antibacterial therapy III. New antimicrobial agents from the quinolones group in clinical trials. Przegl Epidemiol. 2013;67(3):455-460, 557-561. Polish [with English abstract].

3. Arjona A. Nemonoxacin Quinolone antibiotic. Drugs Future. 2009; 3(34):196-203.

4. Adam HJ, Laing NM, King CR, Lulashnyk B, Hoban DJ, Zhanel GG. In vitro activity of nemonoxacin, a novel nonfluorinated quinolone, against 2,440 clinical isolates. Antimicrob Agents Chemother. 2009;53(11):4915-4920. 
5. Chen YH, Liu CY, Lu JJ, King CH, Hsueh PR. In vitro activity of nemonoxacin (TG-873870), a novel non-fluorinated quinolone, against clinical isolates of Staphylococcus aureus, enterococci and Streptococcus pneumoniae with various resistance phenotypes in Taiwan. J Antimicrob Chemother. 2009;64(6):1226-1229.

6. Lauderdale TL, Shiau YR, Lai JF, Chen HC, King CH. Comparative in vitro activities of nemonoxacin (TG-873870), a novel nonfluorinated quinolone, and other quinolones against clinical isolates. Antimicrob Agents Chemother. 2010;54(3):1338-1342.

7. Chotikanatis K, Kohlhoff SA, Hammerschlag MR. In vitro activity of nemonoxacin, a novel nonfluorinated quinolone antibiotic, against Chlamydia trachomatis and Chlamydia pneumoniae. Antimicrob Agents Chemother. 2014;58(3):1800-1801.

8. Chen YH, Liu CY, Ko WC, et al. Trends in the susceptibility of methicillin-resistant Staphylococcus aureus to nine antimicrobial agents, including ceftobiprole, nemonoxacin, and tyrothricin: results from the Tigecycline In Vitro Surveillance in Taiwan (TIST) study, 2006-2010. Eur J Clin Microbiol Infect Dis. 2014;33(2):233-239.

9. Hsu MS, Liao CH, Liu CY, Yang CJ, Huang YT, Hsueh PR. In vitro susceptibilities of clinical isolates of ertapenem-non-susceptible Enterobacteriaceae to nemonoxacin, tigecycline, fosfomycin and other antimicrobial agents. Int J Antimicrob Agents. 2011;37(3):276-278.

10. Chung KP, Tseng SP, Huang YT, Tsai TH, Teng LJ, Hsueh PR. Arrival of Klebsiella pneumoniae carbapenemase (KPC)-2 in Taiwan. JAntimicrob Chemother. 2011;66(5):1182-1184.

11. Yang JC, Lee PI, Hsueh PR. In vitro activity of nemonoxacin, tigecycline, and other antimicrobial agents against Helicobacter pylori isolates in Taiwan, 1998-2007. Eur J Clin Microbiol Infect Dis. 2010;29(11):1369-1375.

12. Liao CH, Ko WC, Lu JJ, Hsueh PR. Characterizations of clinical isolates of clostridium difficile by toxin genotypes and by susceptibility to 12 antimicrobial agents, including fidaxomicin (OPT-80) and rifaximin: a multicenter study in Taiwan. Antimicrob Agents Chemother. 2012;56(7):3943-3949.

13. Lin YC, Huang YT, Tsai PJ, et al. Antimicrobial susceptibilities and molecular epidemiology of clinical isolates of Clostridium difficile in Taiwan. Antimicrob Agents Chemother. 2011;55(4):1701-1705.

14. Lai CC, Tan CK, Lin SH, et al. Comparative in vitro activities of nemonoxacin, doripenem, tigecycline and 16 other antimicrobials against Nocardia brasiliensis, Nocardia asteroides and unusual Nocardia species. J Antimicrob Chemother. 2009;64(1):73-78.

15. Lai CC, Liu WL, Ko WC, et al. Multicenter study in Taiwan of the in vitro activities of nemonoxacin, tigecycline, doripenem, and other antimicrobial agents against clinical isolates of various Nocardia species. Antimicrob Agents Chemother. 2011;55(5):2084-2091.

16. Tan CK, Lai CC, Liao CH, et al. Comparative in vitro activities of the new quinolone nemonoxacin (TG-873870), gemifloxacin and other quinolones against clinical isolates of Mycobacterium tuberculosis. J Antimicrob Chemother. 2009;64(2):428-429.

17. King CHR, Lin L, Leunk R. In vitro resistance development to nemonoxacin for Streptococcus pneumoniae. In: Program and abstracts of 48th Annual Interscience Conference on Antimicrobial Agents and Chemotherapy; October 25-28, 2008; Washington, DC. Abstract C1-1971.
18. Li CR, LiY, Li GQ, et al. In vivo antibacterial activity of nemonoxacin, a novel non-fluorinated quinolone. J Antimicrob Chemother. 2010; 65(11):2411-2415

19. Hsu CH, Lin L, Leunk R, et al. In vivo efficacy of nemonoxacin in a mouse protection model. Abstracts of the Interscience Conference on Antimicrobial Agents and Chemotherapy. 2008;54-54.

20. Hsu CH, Lin L, Leunk R, et al. In vivo efficacy of nemonoxacin in a mouse pulmonary infection model. Abstracts of the Interscience Conference on Antimicrobial Agents and Chemotherapy. 2008:43-43.

21. Lin L, Chang LW, Tsai CY, et al. Dose escalation study of the safety, tolerability, and pharmacokinetics of nemonoxacin (TG-873870), a novel potent broad-spectrum nonfluorinated quinolone, in healthy volunteers. Antimicrob Agents Chemother. 2010;54(1):405-410.

22. Guo B, Zhang J, Yu J, Wu X, Shi Y, Tsai CY. A liquid chromatography-tandem mass spectrometry assay for the determination of nemonoxacin (TG-873870), a novel nonfluorinated quinolone, in human plasma and urine and its application to a single-dose pharmacokinetic study in healthy Chinese volunteers. Biomed Chromatogr. 2012;26(11):1333-1340.

23. Guo B, Wu X, Zhang Y, et al. Safety and clinical pharmacokinetics of nemonoxacin, a novel non-fluorinated quinolone, in healthy Chinese volunteers following single and multiple oral doses. Clin Drug Investig. 2012;32(7):475-486.

24. Chung DT, Tsai CY, Chen SJ, et al. Multiple-dose safety, tolerability, and pharmacokinetics of oral nemonoxacin (TG-873870) in healthy volunteers. Antimicrob Agents Chemother. 2010;54(1):411-417.

25. Chow $\mathrm{CP}$, Tsai $\mathrm{CY}$, Yeh CF, et al. In vitro metabolism and interaction of nemonoxacin (TG-873870) on human hepatic CYP3A4. In: Abstracts of the 47th Annual Interscience Conference on Antimicrobial Agents and Chemotherapy; September 17-20, 2007; Chicago, IL. Abstract A-27.

26. Wright DH, Brown GH, Peterson ML, Rotschafer JC. Application of fluoroquinolone pharmacodynamics. J Antimicrob Chemother. 2000;46(5):669-683.

27. Liang W, Chen YC, Cao YR, et al. Pharmacokinetics and pharmacodynamics of nemonoxacin against Streptococcus pneumoniae in an in vitro infection model. Antimicrob Agents Chemother. 2013;57(7): 2942-2947.

28. Bochud PY, Moser F, Erard P, et al. Community-acquired pneumonia. A prospective outpatient study. Medicine (Baltimore). 2001;80(2): $75-87$.

29. Falguera M, Sacristán O, Nogués A, et al. Nonsevere communityacquired pneumonia: correlation between cause and severity or comorbidity. Arch Intern Med. 2001;161(15):1866-1872.

30. van Rensburg DJ, Perng RP, Mitha IH, et al. Efficacy and safety of nemonoxacin versus levofloxacin for community-acquired pneumonia. Antimicrob Agents Chemother. 2010;54(10):4098-4106.

31. Kosinski MA, Lipsky BA. Current medical management of diabetic foot infections. Expert Rev Anti Infect Ther. 2010;8(11):1293-1305.
Drug Design, Development and Therapy

\section{Publish your work in this journal}

Drug Design, Development and Therapy is an international, peerreviewed open-access journal that spans the spectrum of drug design and development through to clinical applications. Clinical outcomes, patient safety, and programs for the development and effective, safe, and sustained use of medicines are a feature of the journal, which

\section{Dovepress}

has also been accepted for indexing on PubMed Central. The manuscript management system is completely online and includes a very quick and fair peer-review system, which is all easy to use. Visit http://www.dovepress.com/testimonials.php to read real quotes from published authors. 